$\xi=$ 줄

\title{
Management of antero posterior discrepancy using expansion appliances- A case series
}

\author{
Durga Harsha G V ${ }^{1}$, Ananth J ${ }^{2}$, Vijay Krishna B ${ }^{3}$, Padmapriya C V**, Mounica $\mathbf{M}^{5}$,Anoosha $\mathbf{M}^{6}$ \\ ${ }^{I}$ Senior Lecturer, Vishnu Dental College, Bhimavaram \\ ${ }^{2}$ Private practioner, Oman \\ ${ }^{3}$ Senior Lecturer, GSL Dental College, Rajahmundry \\ *4 Professor\& Head, Vishnu Dental College, Bhimavaram \\ ${ }^{5}$ Post graduate student, Vishnu Dental College, Bhimavaram \\ ${ }^{6}$ Senior Lecturer, Vishnu Dental College, Bhimavaram \\ *Corresponding author E-mail:padmapriyacv@vdc.edu.in
}

\begin{abstract}
Antero-posterior deficiencies are one of the major concerns in adolescents especially when presented with class III malocclusion and anterior cross bite. These discrepancies are generally corrected by protraction of maxilla and expansion during growth period. A 16 year old boy and 12 year old girl presented with midface deficiency with anterior cross bite and Class III malocclusion. Antero-posterior expansion was planned with Hyrax and Jack screw appliances respectively. Anterior cross bite and Class III malocclusion was corrected. Majority of these antero posterior discrepancies can be corrected with antero-posterior expansion, hence are of prime importance.
\end{abstract}

Keywords:Skeletal Class III; Growing Patients; Antero Posterior Expansion

\section{Introduction}

Among skeletal class III malocclusions, maxillary retrusion, without mandibular prognathism, was reported to occur in $20 \%$ to $30 \%$ of adult patients (Ellis E, McNamara JA et al 1984). Sue et al found that $62 \%$ of their patients had maxillary skeletal retrusion (Sue GY, Chaconas SJ, Turley PK, Ito JK et al 1987). Bjork and Skieller indicated that maxillary growth was completed in girls by 15 years of age and Savara and Singh et al found that, the maxilla stopped growing by 18 years of age in adolescent boys (Savara BS, Singh IJ et al 1968). It appears that early treatment of Class III dento-skeletal malocclusions should focus on correction of the maxillary deficiency to minimize dentoalveolar effects.

Maxillary retrusion clinically presents as anterior cross bites. It is important to determine whether the anterior cross-bite is a functional displacement of the mandible or a skeletal problem or due to a dental cause. Treatment during the early mixed dentition has been shown to improve maxillary sagittal growth when compared with treatment in the late mixed dentition (Tsai HH. Et al 2001).

The treatment options includes protraction and expansion of maxilla, extractions and /or orthognathic surgery which depends on the growth status of the patient. The treatment options are limited, particularly when growth is completed and the malocclusion is due to the underlying skeletal component. In recent era, Distraction osteogenesis in the premaxilla was considered as an alternative treatment to orthognathic surgery in adult patients with hypoplastic maxilla (Bengi et al 2004).

In the first case, patient presented with deficient maxilla and the patient had crossed pubertal growth spurt. So corticotomy assisted expansion was planned to correct the anterior crossbite as an alternative to surgery. In the second case, patient had growth left, so antero-posterior maxillary expansion was planned. The present case series demonstrates various treatment methods for correcting antero-posterior discrepancies depending on the growth status of the individuals.

\section{Case report 1}

\subsection{Diagnosis}

A 16yr old male patient presented with a chief complaint of forwardly placed lower front teeth. On extra oral examination, patient had concave profile associated with middle third deficiency and straight divergence with competent lips. (Fig.1)

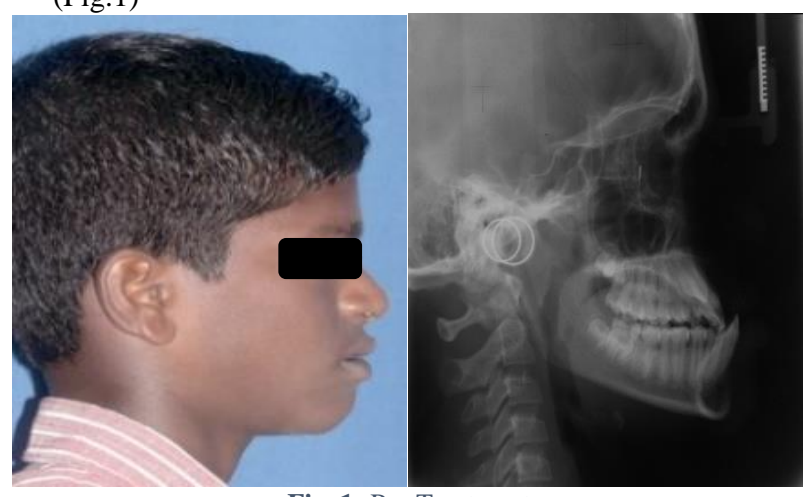

Fig. 1: Pre-Treatment

Intra oral examination revealed class III malocclusion with anterior crossbite with ectopically placed maxillary canines and mild crowding is present in the lower anterior teeth (Fig.2). Mandibular midline deviated $2 \mathrm{~mm}$ to the right and maxillary midline shifted to 
left by $2 \mathrm{~mm}$ and negative overjet and overbite of $-5 \mathrm{~mm}$ and -4 $\mathrm{mm}$ respectively were noted.

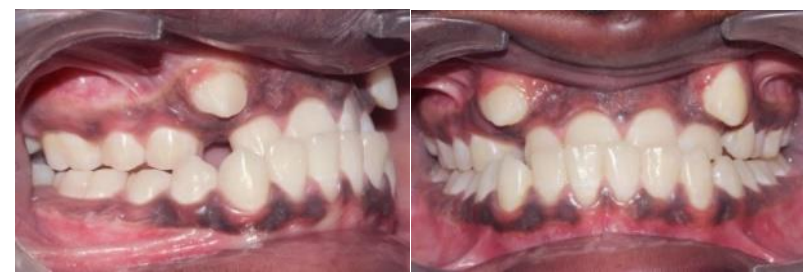

Fig. 2: Pre-Treatment - Class III Malocclusion.

Cephalometric analysis revealed antero-posterior skeletal discrepancies, with an ANB angle of -5.5 degrees, with average to horizontal growth pattern $\left(\mathrm{SN}-\mathrm{Go} \mathrm{Gn}=28^{\circ}, \mathrm{FMA}=24^{\circ}\right)$ and mandibular incisor protrusion $\left(\mathrm{L} 1-\mathrm{NB}=30^{\circ}\right)$. The analysis of panoramic and interproximal radiographs confirmed the presence of full complement of teeth.

Treatment objectives includes improvement of skeletal positioning; correct anterior cross bite; expand the maxilla to ensure a more effective protraction.

\subsection{Treatment plan}

- Phase I: Corticotomy assisted Maxillary antero-posterior expansion with banded hyrax.

- $\quad$ Phase II: Fixed appliance therapy with pre-adjusted edgewise appliance with 0.022 MBT Prescription.

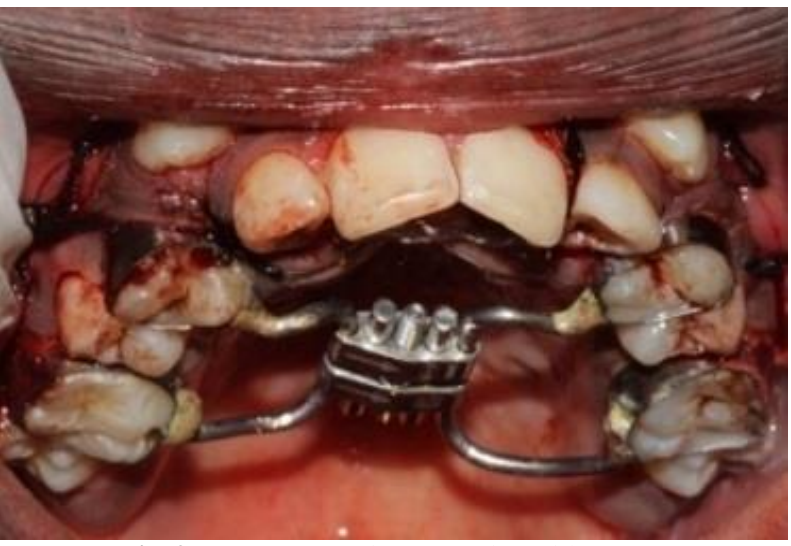

Fig. 3a: Antero-Posterior Expansion with Hyrax.

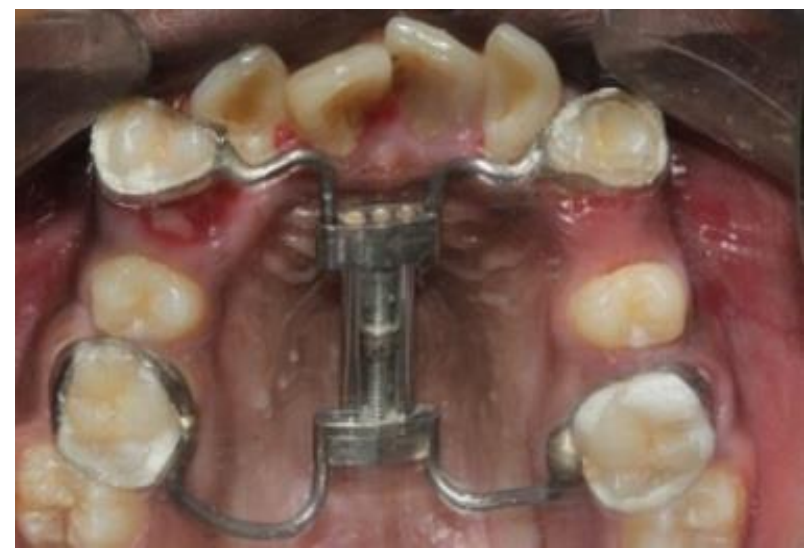

Fig. 3b : After Expansion.

\subsection{Treatment progress}

Initially corticotomy was done and osteotomy cuts were given in between the premolars on both sides and the flap is closed and banded hyrax appliance was placed in the maxillary arch antero posteriorly and occlusal splint is placed in the lower arch (Fig.3a) Hyrax appliance was activated by $1 / 4$ th turn that is 90 degrees twice daily for a period of 3 months and $6 \mathrm{~mm}$ of expansion was achieved (Fig.3b). Nance palatal arch was placed to maintain the expansion space for 6 months during which fixed appliance treatment was continued.

Pre- adjusted Edgewise appliance was placed and 0.014-in, 0.016in, 0.018-in and 0.017x0.025-in NiTi wires were used for alignment and levelling. Distalization of premolars was done by placing open coil spring between the lateral incisors and first premolars to obtain the space for canines, and the canines were brought into occlusion by using 0.019 x0.025 SS wire with 0.014 -inch NiTi piggy back wire in the maxillary arch. Finishing and detailing was done with 0.16 SS wires in the upper and lower arches. Settling elastics were given to improve the intercuspation. The treatment was completed in 36 months (Fig.4) and fixed bonded retainer was placed in the upper and lower arches.

\subsection{Treatment results}
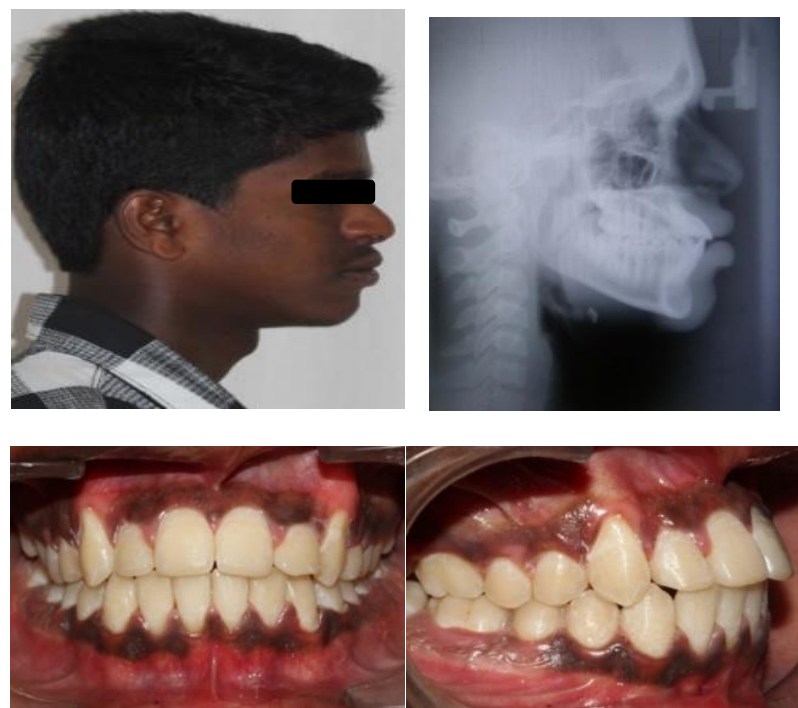

Fig. 4: After 18 Months of Active Treatment.

Anterior crossbite was corrected and ANB angle was improved. The changes were beneficial. Anteroposterior maxilla-mandible relation as well as the inter-relation between maxillary and mandibular incisors was improved. The pre and post treatment cephalometric values were given below.

\begin{tabular}{lll}
\hline Measurements & Before treatment & Aftertreatment \\
\hline SNA & $83^{\circ}$ & $84^{\circ}$ \\
SNB & $88^{\circ}$ & $85^{\circ}$ \\
ANB & $-5^{\circ}$ & $-1^{\circ}$ \\
N per. To A(mm) & $-3.5 \mathrm{~mm}$ & $-2 \mathrm{~mm}$ \\
N per toPog & $11 \mathrm{~mm}$ & $5 \mathrm{~mm}$ \\
FMA & $21^{0}$ & $24^{\circ}$ \\
LAFH(mm) & $64 \mathrm{~mm}$ & $70 \mathrm{~mm}$ \\
Angle of inclination & $87^{0}$ & $89^{\circ}$ \\
Y- axis & $55^{\circ}$ & $58^{\circ}$ \\
Ar-Go-Gn & $125^{\circ}$ & $128^{\circ}$ \\
Nasolabial angle & $83^{\circ}$ & $80^{\circ}$ \\
UI-NA & $29^{0}$ & $39^{\circ}$ \\
UI-NA (mm) & $5 \mathrm{~mm}$ & $10 \mathrm{~mm}$ \\
U1-SN & $109^{\circ}$ & $118^{\circ}$ \\
L1-NB & $30^{\circ}$ & $31^{\circ}$ \\
L1-NB (mm) & $6 \mathrm{~mm}$ & $7 \mathrm{~mm}$ \\
IMPA & $94^{\circ}$ & $95^{\circ}$ \\
Inter incisal angle & $125^{\circ}$ & $106^{\circ}$ \\
Lower lip - E-line $(\mathrm{mm})$ & $5 \mathrm{~mm}$ & $6 \mathrm{~mm}$ \\
\hline
\end{tabular}

\section{Case report 2}

\subsection{Diagnosis}

A 12 year old female patient presented with chief complaint of irregularly placed upper and lower front teeth. 
On extra oral examination patient had middle third deficiency with concave profile and straight divergence with incompetent lips and shallow mento-labial sulcus. On intra oral examination, Angle's class III malocclusion with subdivision on right side with crowding in the upper and lower anterior teeth and anterior cross bite with buccally placed canines in the maxillary arch. Midlines were coinciding, with negative overjet and overbite of $-3 \mathrm{~mm}$ and $-3 \mathrm{~mm}$ respectively (Fig.5).
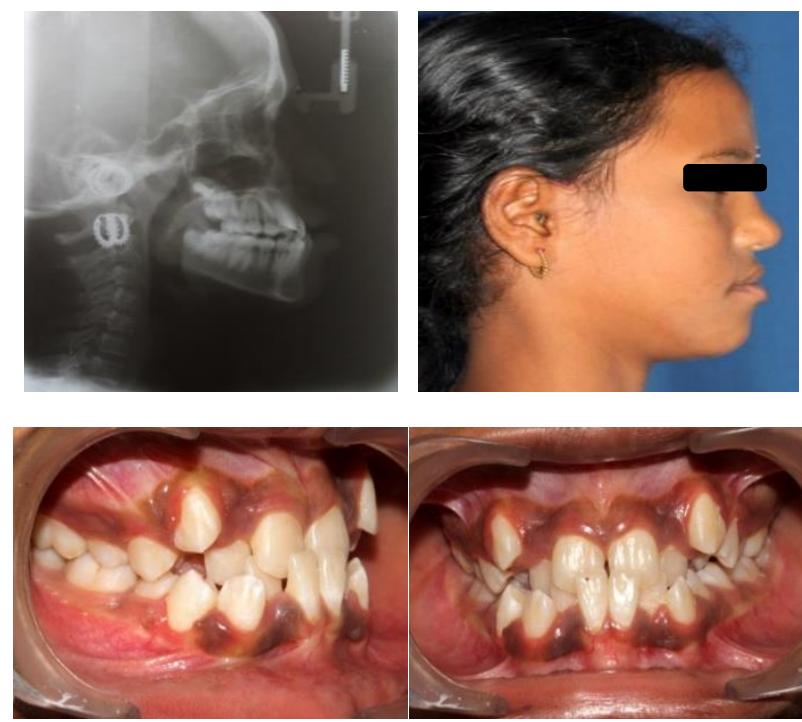

Fig. 5: Pre Treatment - Class III Malocclusion.

Cephalometric analysis demonstrated skeletal class III with ANB angle of $-1^{0}$ with average to vertical growth pattern (SN-Go $\mathrm{Gn}=$ $38^{\circ}$, FMA $=25^{\circ}$ ). Mandible is forwardly placed in relation to the cranial base which resulted in concave profile. Patient was in growing stage with $65-85 \%$ of growth left.

As the patient had crossed the pre-pubertal growth spurt, anteroposterior maxillary expansion was opted in this case rather than facemask therapy.

\subsection{Treatment plan}

- Phase I: Maxillary antero-posterior expansion with jack screw.

- Phase II: Fixed appliance therapy with pre-adjusted edgewise appliance with 0.022 ROTH Prescription.

\subsection{Treatment progress}

Initially, Jackscrew was placed in the maxillary arch anteroposteriorly (Fig.6a) and was activated by 90 degrees once daily for a period of 4 months. After the required expansion was achieved the jackscrew was left in place for retention of the expansion space (Fig.6b). Later, intraoral appliance was removed and fixed appliance was bonded. Extraction of 32 was done. Leveling and alignment was initiated by 0.014 -in NiTi followed by 0.016 -in $\mathrm{NiTi}, 0.017$ x 0.025-in NiTi, 0.018-in stainless steel and 0.019 $\mathrm{x} 0.025$-in stainless steel wire. 0.014-inch Niti piggyback wire placed in the maxillary arch and the canines were brought into occlusion. Finishing and detailing was done with 0.16 SS wires in the upper and lower arches. Settling elastics were given to improve the intercuspation and proper occlusion was established after 13 months of active treatment (Fig.7). Later fixed bonded retainer was placed in the upper and lower arch

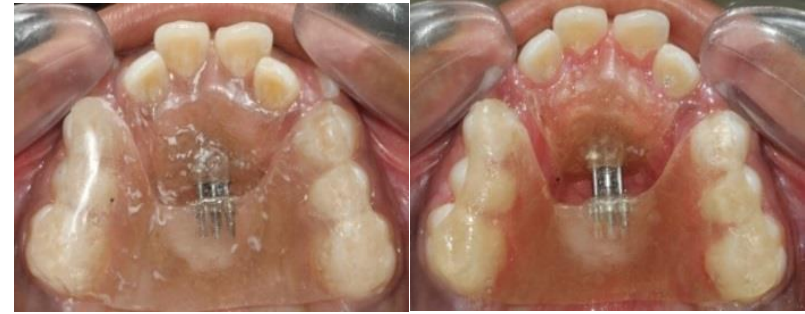

Fig. 6a: Jack screw-before Expansion. Fig 6b: After expansion

\subsection{Treatment results}
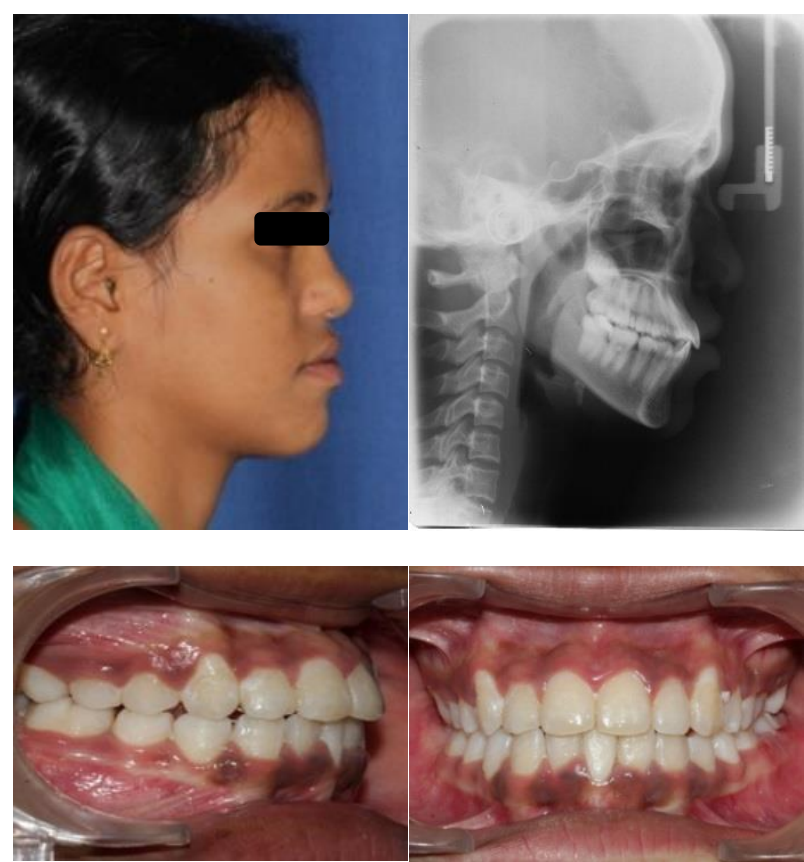

Fig. 7: After 13 Months of Active Treatment.

Anterior cross bite was corrected and ideal overjet and overbite was achieved. Patient esthetics were improved. Changes resulted in a slightly convex profile. The pre- and post- treatment cephalometric values were given below.

\begin{tabular}{lll}
\hline Measurements & Before treatment & Aftertreatment \\
\hline SNA & $76^{\circ}$ & $80^{\circ}$ \\
SNB & $77^{\circ}$ & $78^{\circ}$ \\
ANB & $-1^{\circ}$ & $2^{\circ}$ \\
N per to A(mm) & $-1 \mathrm{~mm}$ & $+1 \mathrm{~mm}$ \\
N per to Pog & $1 \mathrm{~mm}$ & $-2 \mathrm{~mm}$ \\
FMA & $25^{\circ}$ & $23^{\circ}$ \\
Angle of inclination & $93^{0}$ & $89^{\circ}$ \\
Y- axis & $65^{0}$ & $66^{\circ}$ \\
Ar-Go-Gn & $126^{\circ}$ & $125^{\circ}$ \\
Nasolabial angle & $62^{0}$ & $70^{\circ}$ \\
UI-NA & $24^{0}$ & $40^{\circ}$ \\
UI-NA (mm) & $3 \mathrm{~mm}$ & $7 \mathrm{~mm}$ \\
U1-SN & $110^{\circ}$ & $117^{\circ}$ \\
L1-NB & $23^{0}$ & $21^{\circ}$ \\
L1-NB (mm) & $4 \mathrm{~mm}$ & $5 \mathrm{~mm}$ \\
IMPA & $89^{\circ}$ & $86^{\circ}$ \\
Inter incisal angle & $135^{\circ}$ & $123^{\circ}$ \\
Upper lip - E-line (mm) & $2 \mathrm{~mm}$ & $3 \mathrm{~mm}$ \\
Lower lip - E-line (mm) & $5 \mathrm{~mm}$ & $5 \mathrm{~mm}$ \\
\hline
\end{tabular}

\section{Discussion}

Skeletal Class III malocclusion associated with maxillary deficiency requires early intervention as it results in anterior cross bite which was more devastating especially in growing individuals. These changes may compromise facial profile. Early treatment with maxillary protraction and palatal expansion can correct most 
A-P discrepancies in Class III patients (Turley 2007). The best time to commence treatment is early mixed dentition, coincident with eruption of the maxillary permanent central incisors and during pre-pubertal growth spurt.

In the present scenario, the first patient had crossed pubertal growth spurt and the second patient had crossed pre-pubertal growth spurt. So, facemask therapy was not opted and anteroposterior expansion was decided as some amount of growth was remaining in both the cases. Hyrax and Jack screw were placed antero-posteriorly and expansion was done. In the first case corticotomy was done to ensure appropriate amount of protraction as growth was almost completed. Antero-posterior discrepancy and anterior cross bite was corrected in both the cases with more or less skeletal changes and maximum amount of dento-alveolar changes. Upper incisors were proclined and ideal overjet was achieved in both the cases.

Thus early treatment can establish well-balanced occlusal development and proper muscle balance. Early treatment is also directed towards preventing dysplastic growth of both skeletal and the dento-alveolar component. When the patient has not reached pubertal growth spurt, an early intervention is indicated. Palatal expansion is essential as it favors more anterior placement of the maxilla and improves the relation with the mandible, resulting in satisfactory occlusion. In both these cases, patient compliance is good and the anterior crossbite was corrected without the intervention of any orthognathic surgical procedures.

\section{Conclusion}

It should be emphasized that it is very important to correct anterior cross-bites at an early age, reducing the need for long-term orthodontic therapy in the future. For early treatment to be successful, the treatment timing and treatment method are of prime importance. The present appliance design could help general practitioners and paediatric dentists in managing similar type of malocclusions.

\section{References}

[1] Ellis E, McNamara JA. Components of adult Class III malocclusion. J Oral Maxillofac Surg. 1984;42:295-305.

[2] Sue GY, Chaconas SJ, Turley PK, Ito JK. Indicators of Skeleta Class-III Growth. J Dent Res 1987; 66: 345-348)

[3] Turley PK. Treatment of the Class III malocclusion with maxillary expansion and protraction. Semin Orthod. 2007; 1:143-157.

[4] Bengi AO, Gürton A, Okcu KM, Aydintug YS. Premaxillary distraction osteogenesis with an individual tooth-borne appliance. Angle Orthod. 2004;74:420-31.

[5] Tsai HH. Components of anterior crossbite in the primary dentition. ASDC J Dent Child. 2001; 68:27-32.

[6] Dowsing P, Sandler PJ. How to effectively use a $2 \times 4$ appliance. J orthod. 2004; 31:248-58.

[7] Savara BS, Singh IJ. Norms of size and annual increments of seven anatomical measures of maxillae in boys from three to sixteen years of age. Angle Orthod.1968;38:104-20.

[8] Björk A, Skieller V. Growth of the maxilla in three dimensions as revealed radiographically by the implant method. Br J Orthod. $1977 ; 4: 53-64$ 